\title{
Optimization of Inspection Schedule for Hidden-Function Systems
}

\author{
Xiaoli Zou \\ School of Civil Engineering and Architecture, Wuhan Polytechnic University, Wuhan 430023, P. R. \\ China \\ xiaolizou@eyou.com
}

Keywords: Hidden-function system; Inspection of hidden failure; Cost model; Optimal policy

\begin{abstract}
An optimal model for determining minimum-cost inspection schedules for hidden-function systems is presented. The probabilities of the hidden-function system failure as well as the failure of the object the system intends to protect are taken into account to develop this model. Through balancing the cost of multiple failure against the cost of planned inspection, an optimal policy is established which minimizes the expected total cost. As an example, the calculation results for a hidden-function system with Weibull failure distribution are given.
\end{abstract}

\section{Introduction}

The inspection for hidden failure is one of preventive maintenance, which tests the hidden function of a system regularly to prevent the occurrence of a multiple failure. Many protection systems and alarm systems, such as power-system protection relays, electronic security systems, and environmental monitoring equipments, are hidden-function systems. The failures of a hidden-function system are not self-announcing, i.e., they are not evident to the normal operators. The hidden failures are detected only through inspection. Hidden failures itself has no direct adverse effect. However, if the hidden failure is not detected and corrected, once the objects the system intends to protect happen to fail sequentially, it will lead to a multiple failure. The consequence of a multiple failure is often serious. Inspections of the hidden-function system could find and correct the system failure in time so as to decrease the possibility of multiple failure. On the other hand, the cost of preventive maintenance increases with the frequency of inspection. So in developing a preventive maintenance schedule of a hidden-function system, an optimal inspection period should be found to make the best use of the system.

Nakagawa [1] and Ntuen [2] proposed some optimal inspection strategies for hidden failures by using cost model. Wortman et al [3] obtained the inspection time for hidden function system under given system availability. All of these models did not include the failure probability of the object the system intends to protect. In this paper, the probability of the multiple failure is taken into account and an optimal inspection period is determined through balancing the cost of the multiple failure against the cost of inspection.

\section{Optimal Model}

Suppose that $f(t)$ is the probability density function of the hidden-function system failure time $T$. The inspections for hidden failure are carried out only at equally separated moments $\tau, 2 \tau, \ldots, k \tau, \ldots$, and the cost of one inspection is constant $C_{M}$. As shown in Fig. 1, the hidden-function system fails at moment $t,(k-1) \tau<t \leq k \tau$. During the time from the system failure moment $t$ to the next inspection moment $k \tau$, if the object the system intends to protect happens to fail, a multiple failure will occur. The cost of once multiple failure is constant $C_{F}$ 。 Generally, Under the circumstance that the hidden-function system has failed, the random object failure time $S$ can be assumed as exponential distribution with failure rate $\lambda$. 


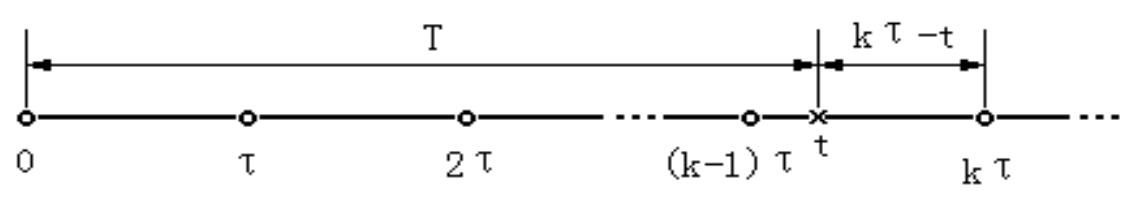

Figure 1. Inspections and failure moments of the hidden-function system

If an inspection finds that the hidden-function system is operational, no more maintenance action is taken until the next inspection. Otherwise, When an inspection find that the system has failed, the system is restored or replace to good as new. Then a new cycle begins. The total cost of one cycle is as

$$
C=C_{M} \sum_{k=1}^{\infty} k \mathrm{I}\{(k-1) \tau<T \leq k \tau\}+C_{F} \sum_{k=1}^{\infty} \int_{(k-1) \tau}^{k \tau} \mathrm{I}\{t \leq T<t+d t, 0<S \leq k \tau-t\} d t
$$

where $\mathrm{I}\{\mathrm{A}\}$ is an indicator function of event $\mathrm{A}$.

So the average total cost of one cycle is give by

$$
\mathrm{E} C=C_{M} \sum_{k=1}^{\infty} k \mathrm{P}\{(k-1) \tau<T \leq k \tau\}+C_{F} \sum_{k=1}^{\infty} \int_{(k-1) \tau}^{k \tau} \mathrm{P}\{t \leq T<t+d t, 0<S \leq k \tau-t\} d t
$$

where

$$
\begin{aligned}
& \begin{aligned}
\sum_{k=1}^{\infty} k \mathrm{P}\{(k-1) \tau<T & \leq k \tau\}=\sum_{k=1}^{\infty} k\{\mathrm{R}[(k-1) \tau]-\mathrm{R}(k \tau)\} \\
= & {[\mathrm{R}(0)-\mathrm{R}(\tau)]+2[\mathrm{R}(\tau)-\mathrm{R}(2 \tau)]+3[\mathrm{R}(2 \tau)-\mathrm{R}(3 \tau)]+\ldots } \\
= & \mathrm{R}(0)+\mathrm{R}(\tau)+\mathrm{R}(2 \tau)+\mathrm{R}(3 \tau)+\ldots=\sum_{k=0}^{\infty} \mathrm{R}(k \tau)
\end{aligned} \\
& \begin{aligned}
\sum_{k=1}^{\infty} \int_{(k-1) \tau}^{k \tau} \mathrm{P}\{t \leq T<t+d t, 0<S \leq k \tau-t\} d t & =\sum_{k=1}^{\infty} \int_{(k-1) \tau}^{k \tau}\left[1-e^{-\lambda(k \tau-t)}\right] f(t) d t \\
& =1-\sum_{k=1}^{\infty} e^{-k \lambda \tau} \int_{(k-1) \tau}^{k \tau} e^{\lambda t} f(t) d t
\end{aligned}
\end{aligned}
$$

In Eq. 3, $\mathrm{R}(t)$ is the reliability function of the hidden-function system, i.e. $\mathrm{R}(t)=\int_{t}^{\infty} f(x) d x$. Substituting Eq. 3 and 4 into Eq. 2

$$
\mathrm{EC}=C_{M} \sum_{k=0}^{\infty} \mathrm{R}(k \tau)+C_{F}-C_{F} \sum_{k=1}^{\infty} e^{-k \lambda \tau} \int_{(k-1) \tau}^{k \tau} e^{\lambda t} f(t) d t
$$

Setting the partial derivative of $\mathrm{EC}$ with respect to $\tau_{\text {zero yields }}$

$$
\frac{\partial \mathrm{EC}}{\partial \tau}=\lambda C_{F} \sum_{k=1}^{\infty} k e^{-k \lambda \tau} \int_{(k-1) \tau}^{k \tau} e^{\lambda t} f(t) d t-\left[C_{M}+C_{F}\left(1-e^{-\lambda \tau}\right)\right] \sum_{k=1}^{\infty} k f(k \tau)=0
$$

The optimal inspection period $\tau^{*}$ can be obtained by solving above equation. Generally, Eq. 6 need to be solved numerically. 


\section{Numerical Example and Conclusions}

Weibull distribution is the most commonly used statistical lifetime model in reliability study. The great experimental and practical data demonstrate that many components and simple systems have Weibull failure distributions. Therefore the failure time $T$ of a hidden-function system is assumed to be two parameter Weibull distribution and its probability density function is

$$
f(t)=\frac{\beta}{\eta}\left(\frac{t}{\eta}\right)^{\beta-1} e^{-\left(\frac{t}{\eta}\right)^{\beta}} \quad t>0
$$

where $\eta$ is the scale parameter; $\beta$ the shape parameter.

Provides $C_{M}=1, C_{F}=300, \lambda=10^{-3}, \beta=2$ and $\eta=500$, the average total cost $\operatorname{EC1}(\tau)$ for the different inspection period $\tau$ are computed according to Eq. 5. The results are shown in Fig. 2. Another curve EC2 $(\tau)$ in Fig. 2 is for $\eta=600$. It is obvious that both curves in Fig. 2 have the lowest points, i.e., there is an optimal inspection period $\tau^{*}$ that minimizes the average total cost $\mathrm{EC}$.

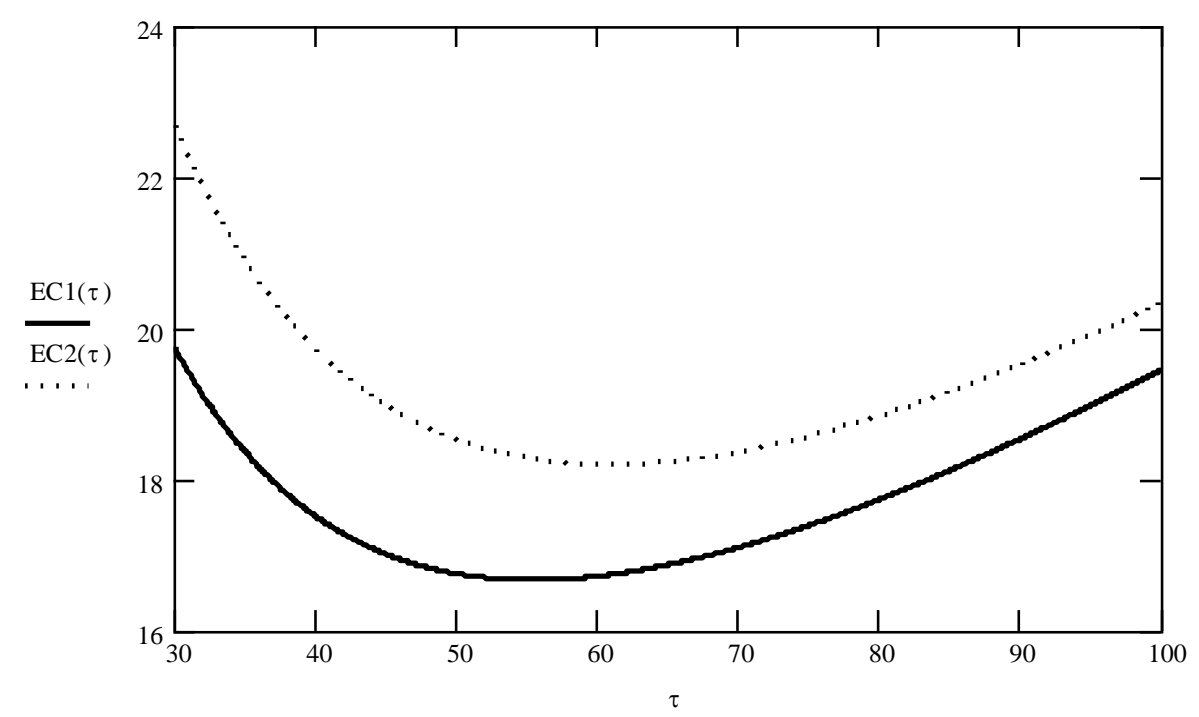

Figure 2. The average total cost for the different inspection period

Substituting Eq. 7 into Eq. 6, the optimal inspection period $\tau^{*}$ can be obtained by solving Eq. 6 . Table 1 shows the computed optimal inspection period for various parameters $\lambda, \beta, \eta$ and cost ratio $C_{F} / C_{M}$. We can find in Table 1 that the optimal inspection periods for two curves in Fig. 2 are 55.339 and 60.674 respectively. 
Table 1 Optimal inspection period for different parameters

\begin{tabular}{cccccccc}
\hline & \multicolumn{3}{c}{$\lambda=10^{-3}, \beta=2$} & & \multicolumn{3}{c}{$\eta=800, \beta=2$} \\
\cline { 3 - 4 } \cline { 7 - 8 }$C_{\mathrm{F}} / \mathrm{C}_{\mathrm{M}}$ & $\eta=400$ & $\eta=500$ & $\eta=600$ & & $\lambda=5 \times 10^{-3}$ & $\lambda=10^{-3}$ & $\lambda=5 * 10^{-4}$ \\
\hline 100 & 86.376 & 96.384 & 106.655 & & 58.577 & 123.212 & 171.549 \\
200 & 60.675 & 67.809 & 74.704 & & 40.237 & 86.378 & 120.845 \\
300 & 49.307 & 55.339 & 60.676 & & 32.435 & 70.355 & 98.771 \\
400 & 42.687 & 47.749 & 52.46 & & 27.871 & 60.676 & 85.179 \\
500 & 38.064 & 42.687 & 46.785 & & 24.815 & 54.206 & 76.214 \\
\hline
\end{tabular}

From Table 1, we can see that the higher the cost of multiple failure is, the smaller the optimal inspection period is. This means frequent inspection requires in order to avoid the high cost of multiple failure. Moreover, the larger the scale parameter of the system failure time or the failure rate of object is, the smaller the optimal inspection period is. This is because that the probability of multiple failure increases with the scale parameter of the system failure time or the failure rate of object. To reduce the possibility of the multiple failure, it requires more inspection.

\section{References}

[1] T. Nakagawa, Periodic and sequential preventive maintenance policies, J. Applied Probability, 23 (1986) 536-542.

[2] C. A. Ntuen, An economic preventive maintenance scheduling model with truncated Gamma function, Reliability Engineering and System Safety, 31 (1991) 31-38.

[3] M. A. Wortman, G. A. Klutke, H. Ayhan, A maintenance strategy for system subjected to deterioration governed by random shocks, IEEE Transactions On Reliability, 43 (1994) 439-445.

[4] F. Beichelt, K. G. Fisher, General failure model applied to preventive maintenance, IEEE

[5] M. Abdel-Hameed, Optimum replacement of systems subject to shocks, J. Applied Probability, 23 (1986) 107-114. 